\title{
Maternal ophthalmic artery Doppler velocimetry in pre-eclampsia in Southwestern Nigeria
}

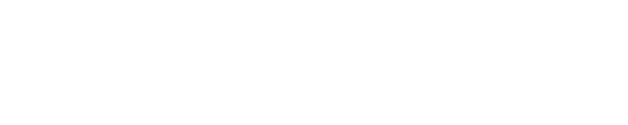

\author{
Richard Busayo Olatunji' \\ Ademola Joseph Adekanmi' \\ Millicent Olubunmi Obajimi' \\ Olumuyiwa Adebola Roberts ${ }^{2}$ \\ Temitope Olumuyiwa Ojo ${ }^{3}$ \\ 'Department of Radiology, \\ ${ }^{2}$ Department of Obstetrics and \\ Gynaecology, University College \\ Hospital, Ibadan, Oyo State, \\ ${ }^{3}$ Department of Community \\ Medicine, Obafemi Awolowo \\ University Teaching Hospital \\ Complex, Ile-Ife, Osun State, Nigeria
}

Background: Pre-eclampsia (PE) poses a serious challenge to maternal and fetal health in Africa. It is associated with hemodynamic changes that may affect the internal carotid/ophthalmic artery circulation with consequent neuro-ophthalmic manifestations. Ophthalmic artery Doppler (OAD) ultrasound is an important tool that can be used to detect hemodynamic changes in PE and monitor its severity. In this study, we evaluated hemodynamic changes on OAD ultrasound in the ophthalmic arteries of pre-eclamptic women and compared these with values in healthy pregnant women.

Methods: OAD parameters, such as, peak systolic velocity, peak diastolic velocity, end diastolic velocity, pulsatility index, and peak ratio, were measured on transorbital triplex ultrasound scan with a 7-10 MHz multifrequency linear transducer in 42 consenting pre-eclamptic patients and 41 pregnant controls matched for maternal age, gestational age, and parity at the Department of Radiology, University College Hospital, Ibadan. Univariate, bivariate, and receiver operating characteristic curve data analyses were performed. $P<0.05$ was considered to be statistically significant.

Results: Mean resistivity index, pulsatility index, and peak systolic velocity were significantly lower in pre-eclamptic patients than in the controls. Mean peak diastolic velocity, end diastolic velocity, and peak ratio were significantly higher in the pre-eclamptic group. The receiver operating characteristic curve showed that the resistivity index (sensitivity $75 \%$, specificity $77.8 \%$ ) could distinguish mild from severe PE while the peak ratio (sensitivity $90.5 \%$, specificity $81.3 \%$ ) could accurately detect PE.

Conclusion: OAD ultrasound can be used to monitor patients with PE for early detection of progression to severe forms before cerebral complications develop. OAD screening of patients at high risk for PE can also detect early changes of hemodynamic derangement.

Keywords: ophthalmic artery, hemodynamic changes, ultrasound, Doppler parameters

\section{Introduction}

Pre-eclampsia (PE) is a pregnancy-related hypertensive disorder occurring usually after 20 weeks of gestational age (GA) that may progress to eclampsia, which is the end point of the disease spectrum if left untreated. ${ }^{1}$ While hypertensive disorders (chronic or pregnancy-induced) complicate $6 \%-30 \%$ of all pregnancies throughout the world ${ }^{2}$ $\mathrm{PE}$ and eclampsia remain the most important hypertensive disorders in pregnancy. The incidence of $\mathrm{PE}$ ranges from $5 \%$ to $10 \%$ worldwide. ${ }^{3} \mathrm{PE}$ is a major cause of maternal mortality and morbidity globally, ${ }^{3}$ and is documented in the literature to be more prevalent in blacks. ${ }^{4,5}$ The prevalence of PE in Nigeria ranges between $2 \%$ and $16.7 \% .^{6,7}$ This condition contributes significantly to fetal wastage and perinatal mortality and morbidity. ${ }^{4,5}$ Even though the pathogenesis of PE is unclear, ${ }^{8}$ and several hypotheses have been proposed. ${ }^{2}$ The most widely accepted is that of endothelial dysfunction leading to defective release of nitric oxide, prostacyclin, and other potent endogenous
Correspondence: Ademola J Adekanmi Department of Radiology, College of Medicine/University College Hospital, University of Ibadan, Queen Elizabeth Road, Ibadan, Oyo State Nigeria Tel +234803354 4856

Email aadekanmi@comui.edu.ng 
vasodilators. ${ }^{7,9,10}$ The resultant widespread vasospasm leads to protean consequences in multiple organ systems. ${ }^{8}$ The diagnostic criteria for PE according to the National High Blood Pressure Education Program in the USA ${ }^{11}$ includes a blood pressure (BP) of more than 140/90 $\mathrm{mmHg}$ with proteinuria $>300 \mathrm{mg}$ in a 24-hour urine sample (or $1+$ on dipstick random urine test) after the 20th week of GA as the minimum criteria. The clinical diagnosis is more accurate in the presence of higher BP and proteinuria levels, persistent visual disturbance, and headache, as well as epigastric pain. Symptomatically, persistent headache and persistent visual disturbance have been documented as sufficient criteria to identify worsening of a pre-eclamptic condition. Visual disturbance is known to occur in $30 \%-100 \%$ of patients with PE. ${ }^{11}$ Eclampsia is the end point of untreated PE. This is defined as seizures that cannot be attributed to any source other than pregnancy in a woman with $\mathrm{PE},{ }^{9}$ and is characterized by abnormalities of intracerebral blood flow. ${ }^{12}$

\section{Imaging}

Magnetic resonance imaging can be safely used to study intracranial blood flow in pregnancy. MRI is however not readily available or cost-effective, and is contraindicated in some patients, such as those with ferromagnetic implants. Other radiological imaging modalities for evaluating the intracranial blood vessels, such as catheter angiography, computed tomographic angiography, and radionuclide imaging, make use of ionizing radiation that is known to be hazardous to the fetus and are therefore contraindicated in pregnancy. ${ }^{13}$ Transcranial Doppler ultrasound is documented as the safest and fastest direct means of studying the intracranial vessels. However, it is not simple to use, has poor spatial resolution, and requires significant technical expertise for accuracy..$^{13}$ The equipment for transcranial Doppler imaging is also not readily available in many developing nations. Doppler ultrasound of the ophthalmic artery has been utilized in the last 2 decades to study the orbital circulation and to assess ocular as well as retrobulbar diseases. ${ }^{14}$ Ophthalmic artery Doppler (OAD) ultrasonography is safe in pregnancy, cheap, readily available, portable, repeatable, and noninvasive. It is employed for evaluation of the intracranial vascular hemodynamics in $\mathrm{PE}^{15}$ due to the fact that the ophthalmic artery has a similar embryologic origin to that of the intracranial vessels. The ophthalmic artery also has anatomical continuity with the intracerebral circulation, being a direct branch of the internal carotid artery. ${ }^{16}$ The lack of bony, fatty, or gaseous structures in the eyeballs makes insonation of the ophthalmic artery by the transorbital route technically feasible.

\section{Ophthalmic Doppler parameters}

The Doppler parameters used for characterizing flow in the ophthalmic artery include the resistivity index (RI), pulsatility index (PI), peak systolic velocity (PSV), peak diastolic velocity (PDV), end diastolic velocity (EDV), and peak ratio. ${ }^{17}$ The peak ratio, which is the ratio of the PDV to the PSV, has been found to be very useful in assessment of ophthalmic artery blood flow in the hypertensive state. ${ }^{18}$ The PI and RI measure the resistance to blood flow in a blood vessel. PI is PSV-EDV/ TAMAX, where TAMAX is time-averaged maximum velocity. The RI, however, measures resistance distal to the point of measurement and is obtained only at an instant in the cardiac cycle. RI is PSV-EDV/PSV. Since visual symptoms and signs are associated with progression of $\mathrm{PE},{ }^{18}$ measurement of OAD pressure is of great value in evaluation of the hemodynamic changes in PE. The RI and PI are preferred to the PSV and EDV in the study of the ophthalmic artery, as both parameters are independent of the angle of insonation, unlike the PSV and EDV. There is also more variability between the PSV and EDV for both eyes, unlike the good correlation for PI and RI values obtained from both eyes. ${ }^{12,18,19}$ The RI is appropriate for evaluating waveforms with high systolic and diastolic flows, as typically seen in opthalmic arteries. It has been reported to be more useful than the PI in the study of the ophthalmic artery, which is more appropriate in vascular beds with mild-to-moderate resistance. ${ }^{20}$

In the developed world, the ophthalmic arteries have been evaluated using Doppler ultrasound in pregnant women with hypertensive disorders such as $\mathrm{PE},{ }^{21-24}$ following the OAD evaluation in pregnant women with PE first described by Hata et $\mathrm{al}^{23}$ in 1995.

However, there is a paucity of information on this subject in this environment despite the fact that pregnancy-induced hypertension contributes significantly to high maternal mortality. ${ }^{1}$ To our knowledge, this study appears to be the first to evaluate the hemodynamics of the ophthalmic artery in PE in this environment. The aim of this study was to document the hemodynamic changes in PE using the OAD ultrasonography technique in singleton pregnancies and compare with those in normotensive pregnant women living in the study region.

\section{Materials and methods Study site and design}

This was a prospective case-control study conducted in the Department of Radiology, University College Hospital (UCH), a tertiary hospital located in Ibadan, Oyo State, Southwestern Nigeria, between June 2013 and September 
2014. Ethical approval for this study was obtained from the University of Ibadan/University College Hospital Ethical Review Committee. Informed consent was obtained and confidentiality of the participants ensured.

\section{Study population}

Pre-eclamptic patients were recruited from the antenatal clinic and labor ward of the UCH after diagnosis was confirmed by elevated BP and proteinuria. The control subjects were normotensive pregnant patients seen in the antenatal clinic at the $\mathrm{UCH}$.

\section{Sample size}

The sample size was estimated using the formula for comparison of means between two groups: ${ }^{25}$

$$
\mathrm{n}=\frac{2 \delta^{2}\left(Z_{\alpha}+Z_{\beta}\right)^{2}}{D^{2}}
$$

where $\mathrm{n}$ is the sample size for each group, $\delta$ is the standard deviation of the outcome variable $(0.2,0.04$, and 0.09 for PI, RI, and peak ratio, respectively), ${ }^{20} \mathrm{Z}_{\alpha}$ is the desired level of statistical significance which at $5 \%=1.96$ and $Z_{\beta}$ is the desired power which at $90 \%=1.28$. $\mathrm{D}$ is the expected minimum difference between the two groups $(0.15,0.05$, and 0.01 for PI, RI and peak ratio, respectively). The sample size for the outcome variables, allowing for an attrition rate of $10 \%$, was 42 subjects and 42 controls. However, a total of 92 pregnant women (42 subjects and 50 controls) were recruited for this study.

\section{Selection criteria}

Inclusion criteria for cases were age of GA $>20$ weeks, elevated $\mathrm{BP} \geq 140 / 90 \mathrm{mmHg}$ on two measurements taken 6 hours apart, and proteinuria $>300 \mathrm{mg}$ in a 24-hour urine sample or at least $1+$ on dipstick random urine test. Inclusion criteria for controls included GA $>20$ weeks, normal $\mathrm{BP}<140 / 90 \mathrm{mmHg}$ on at least two separate antenatal visits 2-4 weeks apart during the index pregnancy, and no proteinuria $(<1+)$ on dipstick random urine test. Exclusion criteria for cases and controls were: a history of diabetes or chronic hypertension; smoking, alcohol abuse, or drug abuse; history of ocular disease; being on medication for hypertension or on corticosteroids; and vasculitis or other vascular disorders that may affect Doppler measurements. Those who did not consent to be involved in the study were also excluded.

\section{Clinical evaluation}

Patients with PE who met the inclusion criteria and consented to the study were recruited as cases and consenting normotensive pregnant women were recruited as controls. A unique study number was assigned to each recruited subject. The control group was matched with the case group for maternal age, GA, and parity.

The GA at recruitment was calculated on the basis of the first trimester ultrasound examination. Where this was not available, GA was estimated from the last menstrual period and confirmed by an obstetric ultrasound performed for inclusion in the study.

BP was measured using an Accoson mercury sphygmomanometer while the patient was at rest. The first and fifth Korotkoff sounds were used to determine the systolic and diastolic BP, respectively. Mean arterial pressure (MAP) was then mathematically derived as $1 / 3$ (systolic BP) $+2 / 3$ (diastolic BP). A urine sample from all recruited patients was checked for proteinuria using an Albustix ${ }^{\circledR}$ test strip.

\section{Ultrasound evaluation}

A General Electric Logiq P5 ultrasound unit with a $10 \mathrm{MHz}$ linear transducer was used to scan the orbit, and OAD parameters were documented. The patient was placed in a supine position with a slight lateral left turn, in accordance with the technique described by Lieb et al. ${ }^{14}$ Both eyes were closed, and after a resting period of 10 minutes, a small amount of acoustic gel was applied on the upper eyelid. The transducer was then gently placed horizontally over the upper eyelid, then tilted upward and downward until the ophthalmic artery was identified using color Doppler flow imaging. Both ophthalmic arteries were insonated, the right before the left. Flow velocity was measured approximately $15 \mathrm{~mm}$ from the optic disc on the medial side of the optic nerve. After recording six consecutive spectral waveforms of similar size and shape, measurements were made on three waveforms. The PI, RI, PSV, PDV, and EDV were measured on each of the three waveforms and an average value from the three measurements was obtained for each parameter. The PSV was the fastest velocity of blood flow in systole, while the EDV was the slowest recorded velocity of blood flow in the diastolic phase of the cardiac cycle. All indices with the exception of the peak ratio were either automatically calculated by the ultrasound equipment or manually estimated by tracing the spectral waveform. The peak ratio was manually calculated as the ratio of the values for the PDV (measured by manual tracing of the spectral waveform at the peak after the protodiastolic notch) and the initial peak (ie, PSV). ${ }^{18}$ Throughout the examination, care was taken to avoid excessive compression of the eyelid with the transducer.

The angle of insonation was kept below $20^{\circ}$, while the filter was set at $50 \mathrm{~Hz}$, the pulse repetition frequency was set 
at $125 \mathrm{kHz}$, and the Doppler sample volume was adjusted to $2 \mathrm{~mm} \cdot{ }^{12,26}$ All patients in the control group were scanned during the third trimester, and all the OAD velocimetry indices (PSV, EDV, PDV, RI, PI, and peak ratio) were also measured sonographically and recorded. Six OAD parameters, ie, PI, RI, PSV, EDV, PDV, and peak ratio, were recorded for each case and control.

\section{Data analysis}

The data were entered and analyzed using Statistical Package for Social Sciences version 17.0 software (SPSS Inc, Chicago, IL, USA). The results are presented using frequency tables or bar charts, and the data distribution is demonstrated in box plots. The PI, RI, PSV, PDV, EDV, and peak ratio values observed in the cases, as well as their age-matched and parity-matched controls, were subjected to analysis using the independent samples (Student's) $t$-test for comparison of the outcome variables. The level of statistical significance was set at $5 \%$. The receiver operating characteristic curve was used to determine the cutoff value of relevant OAD parameters to predict PE and its severity.

\section{Results}

\section{Sociodemographic characteristics of respondents}

Ninety-two patients were recruited into the study (Figure 1).

Forty-two (46\%) had PE and 50 (54\%) were normotensive

\section{Composition of respondents}

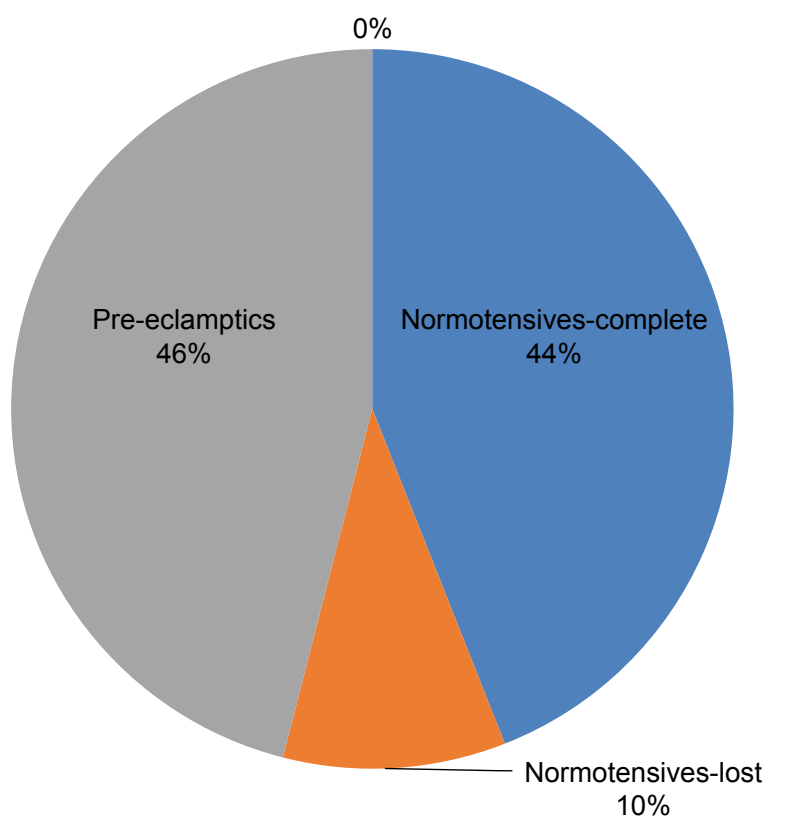

Figure I Pie chart representation of study recruitment shows approximately equal proportions of pre-eclamptic patients and normotensive subjects. pregnant women. Forty-one (82\%) of the normal subjects completed the study. Eighteen (42.9\%) of the pre-eclamptic patients were diagnosed with severe $\mathrm{PE}$ (MAP $\geq 125 \mathrm{mmHg}$ ) and 24 (57.1\%) had mild PE (MAP $<125 \mathrm{mmHg}$ ). ${ }^{27}$

The normotensive patients were recruited at $21-25$ weeks of GA. The mean maternal age was 30.9 \pm 4.2 (range: $23-40$ ) years. Most of the women were nulliparous. The distribution of their systolic BP was between 100 and $120 \mathrm{mmHg}$, diastolic BP was 6-80 $\mathrm{mmHg}$, and MAP was between 70 and $90 \mathrm{mmHg}$. The normotensive patients who attended for the OAD scan (at the third trimester) were aged $2-41$ years, with a mean age of $32.5 \pm 3.9$ years. Their clinicodemographic parameters are as presented in Table 1.

The patients with PE presented most frequently between GA weeks 30 and 34 . They were predominantly nulliparous and usually younger than 35 years of age (Table 2), with a mean age of $32.4 \pm 4.7$ years. Their systolic BP, diastolic BP,

Table I Characteristics of normal patients at the time of OAD scan

\begin{tabular}{|c|c|c|}
\hline Characteristics & Frequency $(n=4 I)$ & Percentage \\
\hline \multicolumn{3}{|l|}{ Age (years) } \\
\hline$<30$ & 8 & 19.5 \\
\hline $30-34$ & 22 & 53.7 \\
\hline $35-39$ & 8 & 19.5 \\
\hline$\geq 40$ & 3 & 7.3 \\
\hline \multicolumn{3}{|l|}{ GA (weeks) } \\
\hline 30 & 4 & 9.8 \\
\hline 31 & 6 & 14.6 \\
\hline 32 & 10 & 24.4 \\
\hline 33 & 9 & 22.0 \\
\hline 34 & 2 & 29.2 \\
\hline \multicolumn{3}{|l|}{ Parity } \\
\hline 0 & 14 & 34.1 \\
\hline I & 17 & 41.5 \\
\hline 2 & 7 & 17.1 \\
\hline$\geq 3$ & 3 & 7.3 \\
\hline \multicolumn{3}{|l|}{ Systolic BP (mmHg) } \\
\hline$<100$ & 2 & 4.9 \\
\hline $100-109$ & 4 & 9.8 \\
\hline $110-119$ & 18 & 43.9 \\
\hline$\geq 120$ & 17 & 41.6 \\
\hline \multicolumn{3}{|c|}{ Diastolic BP (mmHg) } \\
\hline$<60$ & 0 & 0 \\
\hline $60-69$ & 11 & 26.8 \\
\hline 70-79 & 21 & 51.2 \\
\hline$\geq 80$ & 9 & 22.0 \\
\hline \multicolumn{3}{|c|}{ Mean arterial pressure $(\mathrm{mmHg})$} \\
\hline$<70$ & 0 & 0 \\
\hline $70-79$ & 11 & 26.8 \\
\hline $80-89$ & 19 & 46.4 \\
\hline$\geq 90$ & 11 & 26.8 \\
\hline
\end{tabular}

Abbreviations: BP, blood pressure; GA, gestational age; OAD, ophthalmic artery Doppler. 
Table 2 Characteristics of pre-eclamptic patients

\begin{tabular}{|c|c|c|}
\hline Characteristics & Frequency $(n=42)$ & Percentage \\
\hline \multicolumn{3}{|l|}{ Age (years) } \\
\hline$<30$ & 15 & 35.7 \\
\hline $30-34$ & 14 & 33.3 \\
\hline $35-39$ & 10 & 23.8 \\
\hline$\geq 40$ & 3 & 7.2 \\
\hline \multicolumn{3}{|l|}{ GA (weeks) } \\
\hline$<30$ & 8 & 19.0 \\
\hline $30-34$ & 23 & 54.8 \\
\hline $35-39$ & II & 26.2 \\
\hline$\geq 40$ & 0 & 0 \\
\hline \multicolumn{3}{|l|}{ Parity } \\
\hline 0 & 18 & 42.8 \\
\hline 1 & 11 & 26.2 \\
\hline 2 & 5 & 11.9 \\
\hline$\geq 3$ & 8 & 9.1 \\
\hline \multicolumn{3}{|l|}{ Systolic BP (mmHg) } \\
\hline $140-159$ & 7 & 16.6 \\
\hline $160-179$ & 23 & 54.7 \\
\hline $180-199$ & 9 & 21.5 \\
\hline$\geq 200$ & 3 & 7.2 \\
\hline \multicolumn{3}{|c|}{ Diastolic BP (mmHg) } \\
\hline $90-109$ & 25 & 59.5 \\
\hline $110-119$ & 7 & 16.6 \\
\hline $120-139$ & 8 & 19.1 \\
\hline$\geq 140$ & 2 & 4.8 \\
\hline \multicolumn{3}{|c|}{ Mean arterial pressure $(\mathrm{mmHg})$} \\
\hline $110-119$ & 7 & 16.7 \\
\hline $120-139$ & 28 & 66.6 \\
\hline $140-159$ & 5 & 11.9 \\
\hline$\geq 160$ & 2 & 4.8 \\
\hline
\end{tabular}

Abbreviations: BP, blood pressure; GA, gestational age.

and MAP were most commonly in the range of 160-179 $\mathrm{mmHg}, 90-109 \mathrm{mmHg}$, and $120-139 \mathrm{mmHg}$, respectively (Table 2).

The clinicodemographic parameters for the normotensive subjects (controls) were different from those of the preeclamptic patients only with respect to their BP (Figure 2). The mean values for the demographic and clinical characteristics of the normotensive patients and their pre-eclamptic counterparts are presented in Figure 2. The mean parity of the normotensive patients at the time of OAD measurement was $1.0 \pm 1.1$. This was not significantly different from the mean parity of $1.2 \pm 1.4$ for the pre-eclamptic patients $(P=0.87)$.

\section{Comparison of OAD parameters between pre-eclamptic and normotensive patients}

The mean values for the RI, PI, and peak ratio were significantly different between the pre-eclamptic patients and the controls, as shown in Table 3. The RI and PI mean values



Figure 2 Bar chart representation of the mean values of clinicodemographic characteristics of the normotensive and pre-eclamptic participants in the study. Note the similarity in the maternal and gestational ages of the pre-eclamptic patients and the normotensive patients.

Abbreviations: BP, blood pressure; PE, pre-eclampsia.

were significantly lower in the pre-eclamptic group than in the normotensive patients. The mean value of the peak ratio was also significantly higher in the pre-eclamptic patients than in the control group.

Similarly, the PDV of both ophthalmic arteries was significantly higher in the pre-eclamptic group. The EDV was marginally higher in the pre-eclamptic patients than in the control group, but this slight difference was of no statistical significance. The PSV in the left and right ophthalmic arteries, although lower in the pre-eclamptic group, also showed no statistically significant difference (Table 3 ).

The box plot is used to show the data distribution and the data median, as a measure of central tendency, and to present comparisons. Only the box plots of selected right ophthalmic artery blood flow measures are presented in Figure 3A-C since there was no statistically significant difference in $\mathrm{OAD}$ metrics between the right and the left (Table 3). The right RI shows a positively skewed distribution in the pre-eclamptic group while a normal (symmetric) distribution was seen in the control group (Figure 3A). There was a normal distribution of right PI measurements in the pre-eclamptic patients and the control subjects, as shown in Figure 3B. The distribution of the right peak ratio in the normal patients was positively skewed, as shown by the box plot in Figure 3C. However, a normal distribution was seen in the pre-eclamptic patients. The control subjects showed a positively skewed distribution for their right EDV measurements (Figure 3D), while a normal distribution was seen in the pre-eclamptic group.

\section{Comparison of BP and OAD metrics in mild and severe PE}

When compared with values in those with mild $\mathrm{PE}$, mean systolic BP, diastolic BP, and MAP were significantly higher in patients with severe PE. The systolic BP was 
Table 3 Comparison of mean values of OAD parameters between the pre-eclamptic group and follow-up control group

\begin{tabular}{|c|c|c|c|c|}
\hline Variable & $\begin{array}{l}\text { Pre-eclamptic } \\
(n=4 I)\end{array}$ & $\begin{array}{l}\text { Control (2nd US) } \\
(n=4 I)\end{array}$ & $t$-test & $P$-value \\
\hline \multicolumn{5}{|l|}{ Right RI } \\
\hline$($ Mean $\pm S D)$ & $0.62 \pm 0.10$ & $0.70 \pm 0.10$ & -3.53 & $0.00 *$ \\
\hline \multicolumn{5}{|l|}{ Left RI } \\
\hline$($ Mean \pm SD $)$ & $0.63 \pm 0.83$ & $0.70 \pm 0.08$ & -4.20 & $0.00 *$ \\
\hline \multicolumn{5}{|l|}{ Right PI } \\
\hline$($ Mean $\pm S D)$ & $1.2 \pm 0.50$ & $1.60 \pm 0.40$ & -3.12 & $0.00 *$ \\
\hline \multicolumn{5}{|l|}{ Left PI } \\
\hline$($ Mean $\pm S D)$ & $1.15 \pm 0.34$ & $1.54 \pm 0.48$ & -4.24 & $0.00 *$ \\
\hline \multicolumn{5}{|c|}{ Right PSV (cm/sec) } \\
\hline$($ Mean \pm SD $)$ & $28.16 \pm 9.15$ & $32.10 \pm 12.30$ & -1.63 & 0.11 \\
\hline \multicolumn{5}{|l|}{ Left PSV $(\mathrm{cm} / \mathrm{sec})$} \\
\hline$($ Mean $\pm S D)$ & $29.77 \pm 10.69$ & $33.88 \pm 12.22$ & -1.63 & 0.11 \\
\hline \multicolumn{5}{|c|}{ Right PDV (cm/sec) } \\
\hline$($ Mean \pm SD) & $22.56 \pm 7.13$ & $19.06 \pm 6.82$ & 2.28 & $0.03 *$ \\
\hline \multicolumn{5}{|c|}{ Left PDV (cm/sec) } \\
\hline$($ Mean \pm SD) & $25.43 \pm 8.73$ & $19.85 \pm 7.34$ & 3.15 & $0.00 *$ \\
\hline \multicolumn{5}{|c|}{ Right EDV (cm/sec) } \\
\hline$($ Mean \pm SD $)$ & $9.85 \pm 3.31$ & $8.76 \pm 4.00$ & 1.35 & 0.18 \\
\hline
\end{tabular}

Note: $*<0.05$ is significant.

Abbreviations: SD, standard deviation; RI, resistivity index; PI, pulsatility index; PSV, peak systolic velocity; PDV, peak diastolic velocity; EDV, end diastolic velocity; OAD, ophthalmic artery Doppler; US, ultrasound.

A

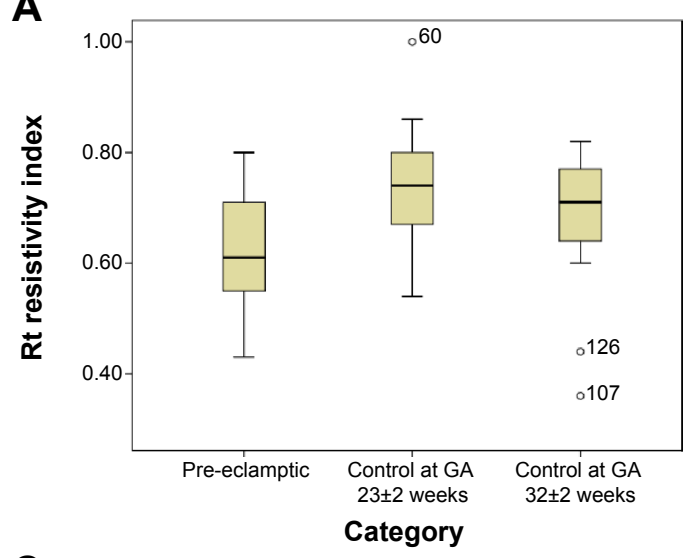

C

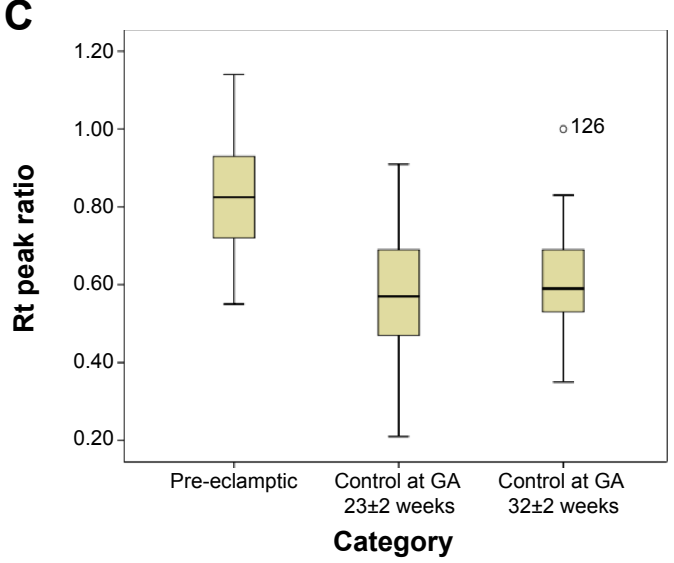

B

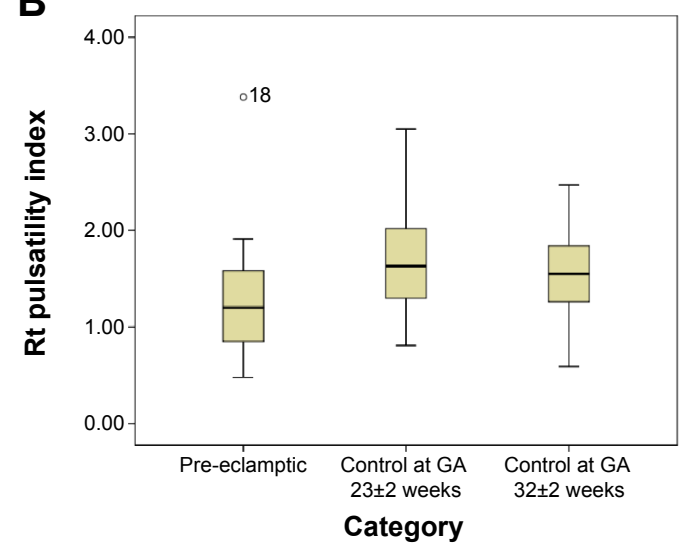

D



Figure 3 Box plots for right RI, PI, PR, and EDV in control subjects at GA of $23 \pm 2$ weeks $(n=50)$, control subjects at GA of $32 \pm 2$ weeks ( $n=4 I$ ) and pre-eclamptic patients $(\mathrm{n}=42)$. (A) Box plots for right RI showing a positively skewed distribution in pre-eclamptics. Note that pre-eclamptic patients have lower RI values. (B) Box plots for the right $\mathrm{PI}$ showing a normal distribution in all the groups. Note that pre-eclamptic patients have lower PI values. (C) Box plots for the right PR showing a positively skewed distribution in control subjects. Note that pre-eclamptic patients have higher PR values. (D) Box plots for right EDV. A positively skewed distribution is shown in the control subjects. Abbreviations: GA, gestational age; PR, peak ratio; RI, resistivity index; EDV, end diastolic velocity; PI, pulsatility index; Rt, right. 
Table 4 Comparison of BP and OAD metrics between patients with mild and severe pre-eclampsia

\begin{tabular}{|c|c|c|c|c|}
\hline Variable & Mild PE $(n=24)$ & Severe PE $(n=18)$ & t-test & $P$-value \\
\hline \multicolumn{5}{|l|}{$\mathrm{SBP}(\mathrm{mmHg})$} \\
\hline Mean \pm SD & $|59.58 \pm 7.5|$ & $|82.78 \pm 23.2|$ & 4.60 & $0.00 *$ \\
\hline \multicolumn{5}{|l|}{$\mathrm{DBP}(\mathrm{mmHg})$} \\
\hline Mean \pm SD & $99.17 \pm 4.08$ & $118.89 \pm 14.10$ & 6.52 & $0.00 *$ \\
\hline \multicolumn{5}{|l|}{ MAP $(\mathrm{mmHg})$} \\
\hline Mean \pm SD & $1 \mid 9.31 \pm 3.68$ & $140.19 \pm 16.03$ & 6.19 & $0.00 *$ \\
\hline \multicolumn{5}{|l|}{ Right RI } \\
\hline Mean \pm SD & $0.66 \pm 0.87$ & $0.59 \pm 0.74$ & -2.44 & $0.02 *$ \\
\hline \multicolumn{5}{|l|}{ Right PI } \\
\hline Mean \pm SD & $1.24 \pm 0.38$ & $1.02 \pm 0.23$ & -2.16 & $0.04 *$ \\
\hline \multicolumn{5}{|c|}{ Right PSV (cm/sec) } \\
\hline Mean \pm SD & $28.44 \pm 8.68$ & $27.80 \pm 9.99$ & -0.22 & 0.83 \\
\hline \multicolumn{5}{|c|}{ Right PDV (cm/sec) } \\
\hline Mean \pm SD & $22.62 \pm 6.48$ & $22.95 \pm 8.09$ & 0.31 & 0.76 \\
\hline \multicolumn{5}{|c|}{ Right EDV (cm/sec) } \\
\hline Mean \pm SD & $9.51 \pm 2.93$ & $10.31 \pm 3.79$ & 0.77 & 0.44 \\
\hline Right PR & $0.81 \pm 0.18$ & $0.84 \pm 0.12$ & 0.68 & 0.50 \\
\hline
\end{tabular}

Note: $* P<0.05$ is significant.

Abbreviations: BP, blood pressure; SBP, systolic blood pressure; DBP, diastolic blood pressure; MAP, mean arterial pressure; SD, standard deviation; RI, resistivity index; PI, pulsatility index; PSV, peak systolic velocity; PDV, peak diastolic velocity; EDV, end diastolic velocity; OAD, ophthalmic artery Doppler; PR, peak ratio; PE, pre-eclampsia.

$182.78 \pm 23.21 \mathrm{mmHg}$ in subjects with severe PE and $159.58 \pm 7.51 \mathrm{mmHg}$ in those with mild PE, with $P$-values of $<0.001$. Diastolic BP and MAP values are as shown in Table 4 and Figure 4A. However, the RI and PI values were significantly lower in patients with severe PE, with $P$-values of 0.02 and 0.04 , respectively (Table 4 , Figure $4 \mathrm{~B}$ and $\mathrm{C}$ ).

\section{Utility of OAD parameters to predict PE and its severity}

The PDV, EDV, and peak ratio are useful to varying extents in predicting a pre-eclamptic state as determined by the receiver operating characteristic curve (Figure 5A-D). The peak ratio shows the highest sensitivity (90.5\%) and specificity (81.3\%) for this purpose when the cutoff value was 0.72 (confidence interval $0.84-0.96 ; P=0.00)$. A mild degree of PE can be predicted by the RI (Figure 5D) with a sensitivity and specificity of $75 \%$ and $77.8 \%$, respectively, when the cutoff value of 0.61 is used (confidence interval $0.54-0.88 ; P=0.02$ ). None of the OAD parameters had an area under the curve significant enough to be of any use to predict severe PE.

\section{Discussion}

$\mathrm{PE}$ is a major cause of maternal mortality, and successful prevention of its attendant morbidity and mortality rests on the tripod of accurate prediction of onset, accurate prediction of progression, and its prevention ab initio. There is no known intervention of any significant impact to prevent PE.
Research should therefore be focused on improving the ability to predict its occurrence and complications. Transorbital Doppler sonography is the main noninvasive method used to quantify perfusion of the ophthalmic artery in pregnant women.

This study documented the pattern of OAD parameters among patients with PE and compared these values with those of a normotensive control group. The mean age of the subjects in this study was $32.5 \pm 3.9$ years and $32.4 \pm 4.7$ years in the $\mathrm{PE}$ and normotensive groups, respectively. These values are higher than those from studies in other climes, which recorded mean maternal ages of $26.7 \pm 4.9$ years, $24.4 \pm 6.6$ years, and $25.7 \pm 1.7$ years. ${ }^{13,16,28-12,16,26}$ However, they are consistent with data on PE from this environment. ${ }^{428-30}$ The higher mean age of pregnant women in this study may partly explain the higher burden of PE in this locality, this agrees with the study by Ananth et al that documented increased PE among 30-34 year old women in the USA. ${ }^{31}$

The mean GA of the pre-eclamptic subjects in this study was $32 \pm 2$ weeks, which is similar to the mean GA of preeclamptic patients reported by other researchers. ${ }^{21,25,32-34}$ This suggests that early-onset PE may be the more common subtype of PE in the study populations. Recent data from the western world indicate that black women and older age are strongly associated with early-onset PE. ${ }^{35}$ The racial and age profile of the pre-eclamptic patients in this study fits this description. On the other hand, the relatively high mean GA of the pre-eclamptic study participants may 
A

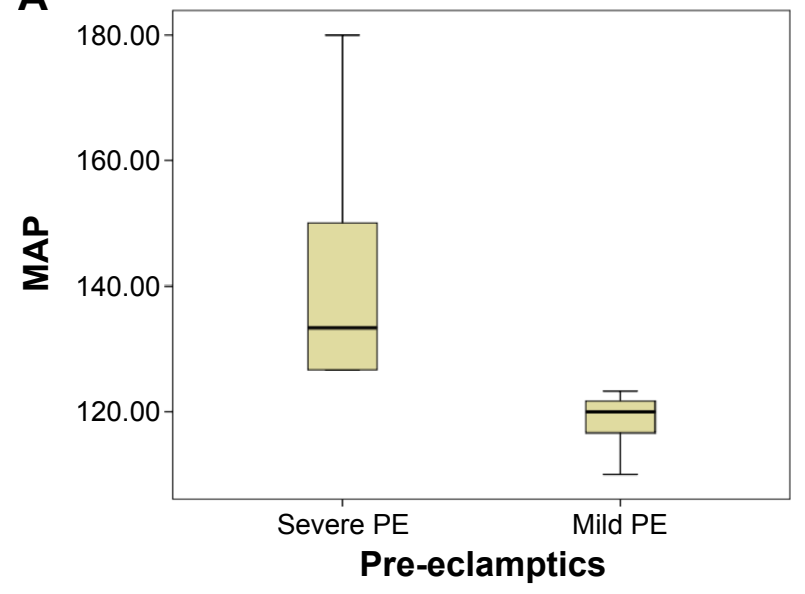

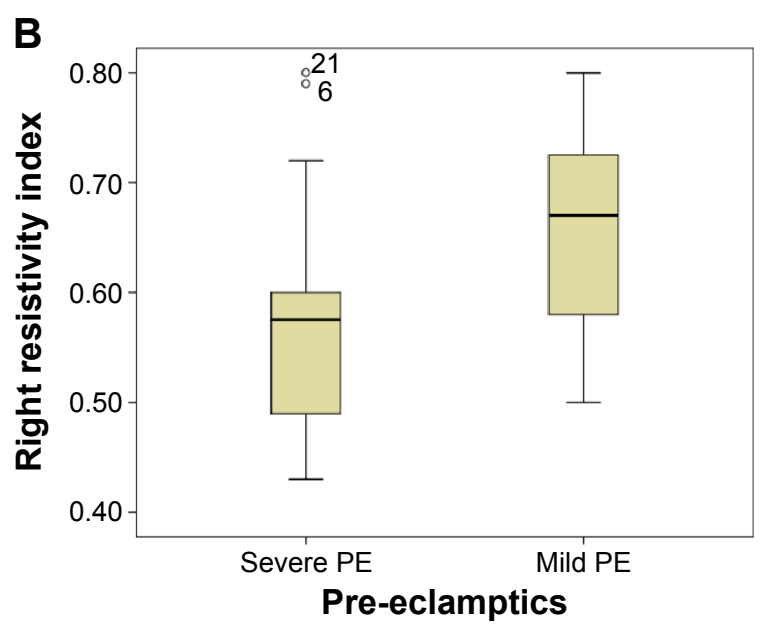

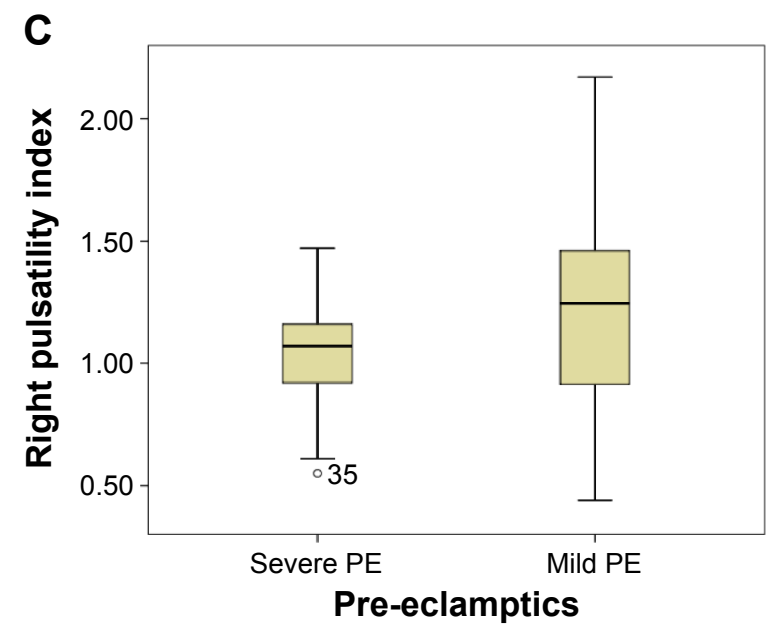

Figure 4 Box plots of MAP, RI, and PI among the pre-eclamptic subjects (mild pre-eclampsia [n=24], severe pre-eclampsia [n=l8]). (A) Box plots for mean arterial pressure in those with mild pre-eclampsia and in those with severe pre-eclampsia. (B) Box plots for right RI among those with mild pre-eclampsia and those with severe pre-eclampsia. (C) Box plots for right PI among those with mild pre-eclampsia and those with severe pre-eclampsia.

Abbreviations: PE, pre-eclampsia; RI, resistivity index; PI, pulsatility index; MAP, mean arterial pressure.

also reflect either delayed presentation of patients or subtle late onset of PE. Ugwu et $\mathrm{al}^{36}$ reported 34-36 weeks as the commonest range of GA at which patients with PE presented over a 4-year period in Enugu, Nigeria. The contribution of delayed onset of PE to this observed pattern is a consideration for future research.

There was no significant difference in the values of the OAD parameters on the right and left, in agreement with observations from most previous studies; this may suggest no racial differences in the variability of OAD pressure in both eyes among native black Africans and other races. ${ }^{14,19,33,37}$

The statistically significant difference in OAD parameters between the control and the pre-eclamptic groups found in this study is similar to the findings of several research groups elsewhere. ${ }^{14,19,23,33,38-42}$ The lower mean values for RI and PI suggest a much lower impedance to blood flow in the ophthalmic artery of patients with PE. This is beyond what would be accounted for by the normal peripheral vasodilatation associated with increasing GA in healthy pregnant women. This assertion is further reinforced by the finding of lower PSV values in the preeclamptic group when compared with the control group. Whereas the decrease in impedance indices (RI and PI) was accompanied by an increase in PSV values for normal progression in healthy pregnancies, it was accompanied by a decrease in PSV values in pregnancies complicated by PE. This decrease in PSV values, although not statistically significant, was observed in mild PE as well as in severe PE. There was also a statistically insignificant trend toward an increase in PSV values from mild-to-severe PE. However, the observations in this study as regards the trend of PSV in normotensive and pre-eclamptic patients are at variance with reports in the literature. ${ }^{20,27,40,42}$ Takata et al ${ }^{33}$ found no difference in PSV between patients with mild PE and those with severe PE, a finding that is closer to that in this present study. The smaller sample size used in most 
A

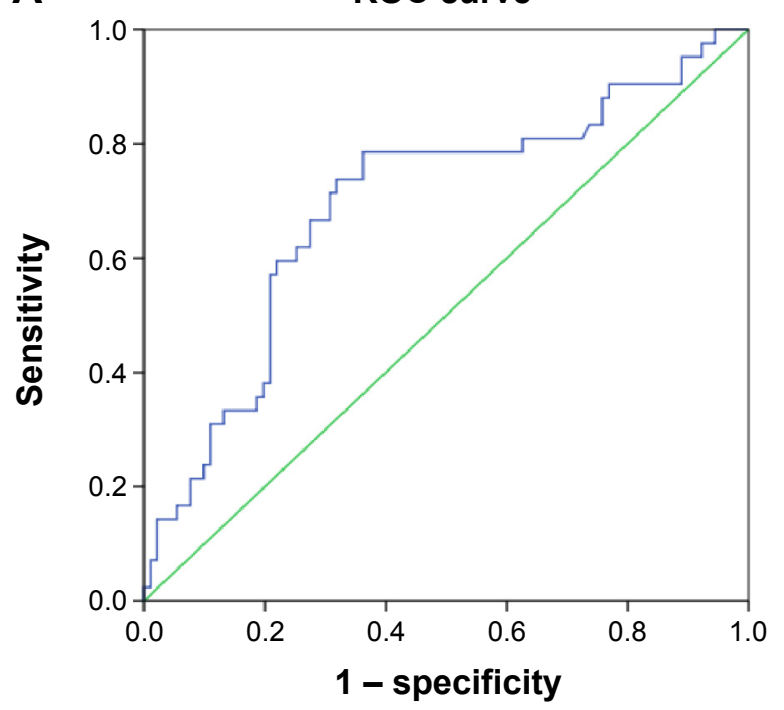

C

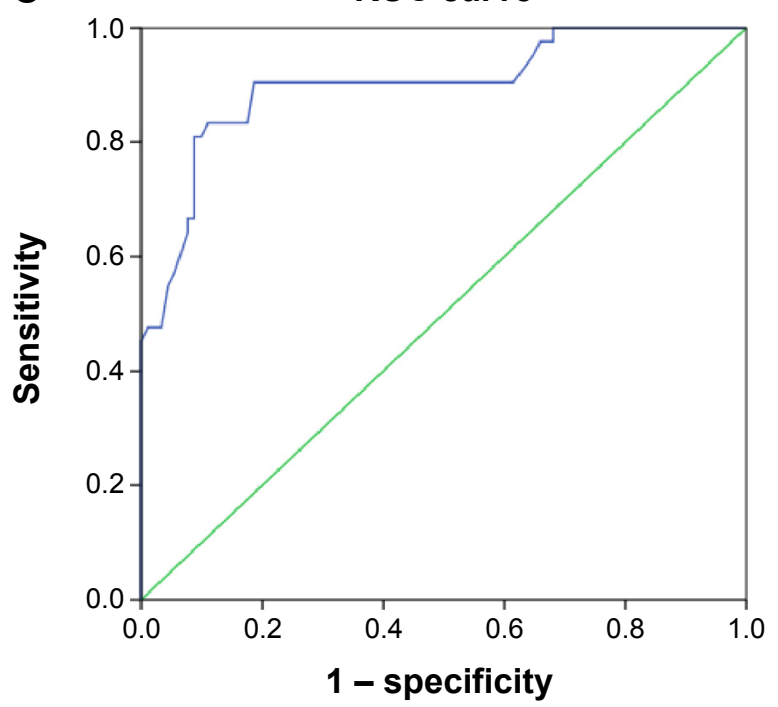

B

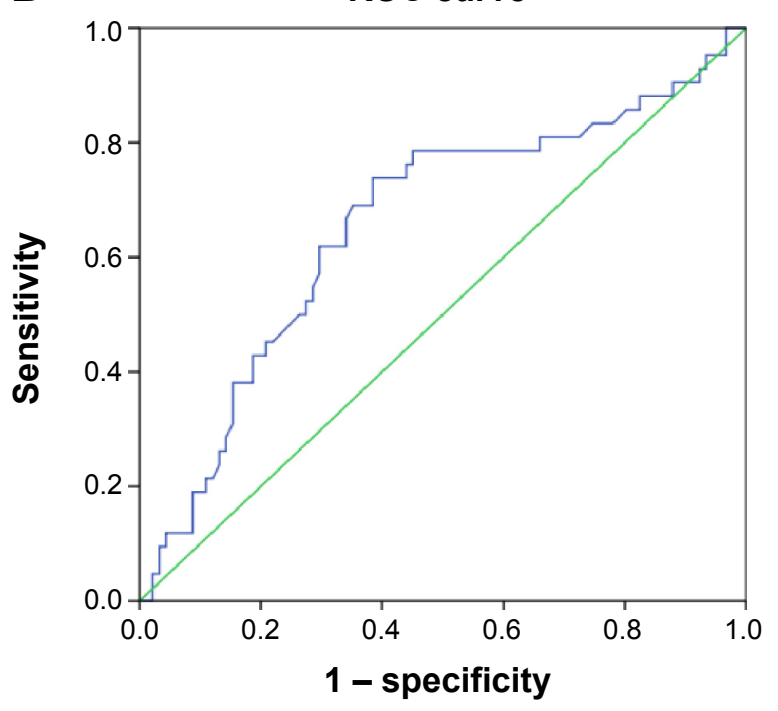

D

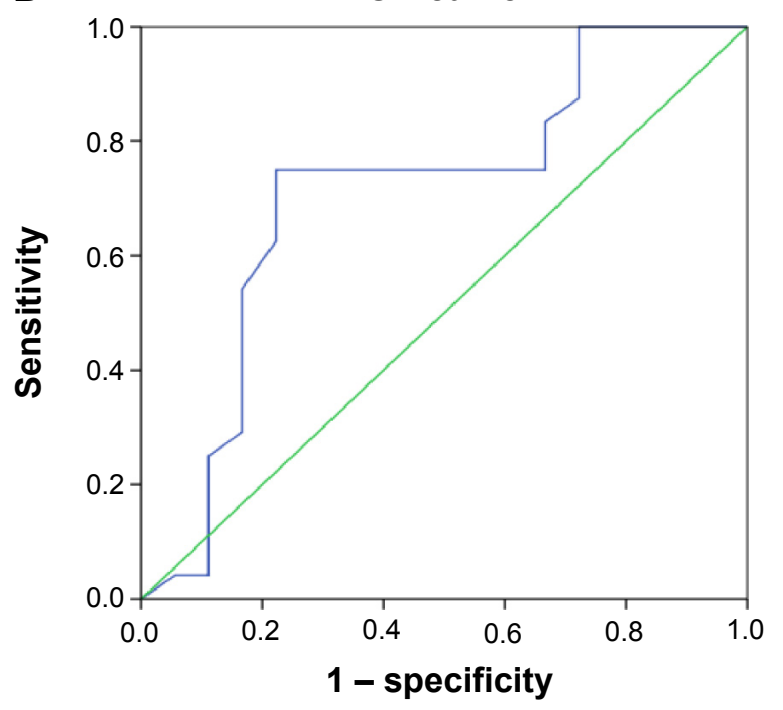

Figure 5 ROC curves for OAD parameters to predict pre-eclampsia and its severity. (A) ROC curve for PDV to predict pre-eclampsia (AUC 0.69; $95 \%$ Cl 0.59-0.79). (B) ROC curve for EDV to predict pre-eclampsia (AUC 0.657; 95\% Cl 0.55-0.76). (C) ROC curve for PR to predict pre-eclampsia (AUC 0.900; 95\% Cl 0.84-0.96). (D) ROC curve for RI to predict mild pre-eclampsia (AUC 0.709; 95\% Cl 0.54-0.88).

Abbreviations: $\mathrm{AUC}$, area under the curve; $\mathrm{Cl}$, confidence interval; ROC, receiver operating characteristic; PR, peak ratio; RI, resistivity index; PDV, peak diastolic velocity; EDV, end diastolic velocity; OAD, ophthalmic artery Doppler.

of these previous studies with discordant results coupled with differences in the study population, instruments, and sonographic techniques used may be partly responsible for the variation in findings.

The observed reduction in mean values for PSV in pre-eclamptic patients may be the key to understanding the mechanism of the characteristic elevation of peak ratio in these groups of patients. Nakatsuka et $\mathrm{al}^{18}$ were the first researchers to describe the peak ratio, and they stated clearly that the "mechanism of elevation of the peak ratio in PE was not clarified" in their study. The elevation of peak ratio may not only be due to the rise in PDV but also result from the reduction in PSV of PE patients as observed in this present study. The fact that the peak ratio did not change significantly between mild and severe PE attests to this ( $t=-1.22, P=0.23)$. If both PSV and PDV increased in patients with $\mathrm{PE}$ as reported by many studies, the peak ratio, being a ratio of these two velocities, should not have changed appreciably in contradistinction to the general belief of rising PR in PE patients. Furthermore, because the PSV and PDV increased in normal pregnancy from the second to third trimester, the peak ratio remained largely constant throughout pregnancy, as consistently reported in the literature. ${ }^{12,18,23,33,43}$ This is why the mild rise in PSV 
and PDV may be responsible for the lack of a statistically significant change in peak ratio between mild and severe PE $(t=-1.22, P=0.23)$ in this study.

As documented in the literature, diastolic flow is higher in patients with $\mathrm{PE}$ than in those with normal pregnancies. ${ }^{12,20,23,26,33,43}$ It is also uniformly agreed that diastolic flow is higher in severe PE than in mild PE. ${ }^{23,34}$ This same finding for PDV and EDV observed in this study between these groups of patients is therefore in concordance with reports from previous studies.

This study also determined the cutoff values of some OAD parameters to predict the presence of PE. A peak ratio value of 0.72 and above can predict $\mathrm{PE}$ with a high sensitivity (90.5\%) and specificity (81.3\%). Other useful parameters for this purpose as determined in this study are the PDV (cutoff $21.33 \mathrm{~cm} / \mathrm{sec}$, sensitivity $71.4 \%$, and specificity $69.2 \%$ ) and EDV (cutoff $7.78 \mathrm{~cm} / \mathrm{sec}$, sensitivity $71.4 \%$, and specificity $61.5 \%)$.

A cutoff value for RI of 0.61 can also discriminate mild from severe PE with a sensitivity of $75 \%$ and a specificity of $78.8 \%$ ( $P=0.02)$. In other words, RI values below 0.61 suggest progression to severe PE. This is similar to the cutoff value of 0.657 (sensitivity $73.3 \%$, specificity $88.8 \%$ ) defined by de Oliveira et $\mathrm{al}^{34}$ in their Brazilian population. It is also consistent with the finding of Barbosa et $\mathrm{al}^{44}$ who associated RI values of $<0.56$ with clinical evidence of posterior reversible encephalopathy syndrome in Brazil.

In contrast with the report of de Oliveira et al, ${ }^{34} \mathrm{PI}$ and peak ratio were not determined to be useful for distinguishing mild from severe PE by the receiver operating characteristic curves obtained in this present study. This discrepancy may be due to the fact that a MAP value of $125 \mathrm{mmHg}$ was used to define severe PE in this study. A MAP value of $125 \mathrm{mmHg}$ is reported to be more accurate in defining severe PE than both systolic and diastolic BP. ${ }^{27}$ This study therefore used the MAP benchmark in accordance with current recommendations. ${ }^{27,45}$

The identification of women at low risk for developing severe PE by making use of some of the derived cutoff values in this study may have clinical benefits. It may improve the confidence of care providers in adopting conservative management of such pregnancies aimed at enhancing fetal maturation before delivery. This will result in a better pregnancy outcome for mother and child.

Also, the cutoff values can be used to identify preeclamptic women at higher risk of developing cerebral complications, and should encourage intensive therapy aimed at saving the life of the mother. This will have a great impact in reduction of maternal mortality, which is currently a big challenge in Nigeria and many other developing countries. Future studies will be required in different populations to further ascertain how well OAD parameters can predict complications of PE and pregnancy outcomes.

The effects of pharmacological agents in modulating OAD parameters may be evaluated in future research with the ultimate goal of arresting progression to eclampsia and also to assess the effectiveness of current pharmacological therapies.

It is important to state that loss to follow-up in our control group was more than the $10 \%$ rate allowed for in the estimation of sample size. However, an attrition rate of $18 \%$ as recorded in this study is still below the $20 \%$ limit allowed for in statistics. The inferences drawn from this study are therefore unlikely to be affected to any significant extent by this higher than anticipated attrition rate.

Finally, this study did not determine the relationship between OAD parameters and severity of the patient's neurological symptoms as it was not a research question at the inception of the study. This is a limitation of the study and a research question for future studies.

\section{Recommendations}

OAD ultrasonography should be incorporated into the routine evaluation of pregnant women during antenatal care, especially those determined to be at high risk of PE on clinical grounds. These include patients younger than 18 years of age and those over 35 years of age, nulliparous patients, patients with a past history of PE, and patients with new partners. Patients who present with mild PE should undergo baseline OAD ultrasonography in order to determine their risk status as pertains to progression to severe forms of the disease (severe PE and eclampsia). Further research in different parts of Nigeria and among native African population is needed to validate some of the observations in this study which are at variance with studies from other races.

\section{Conclusion}

This study has shown that RI and PI (impedance indices) were reduced in pre-eclamptic patients when compared with healthy pregnant women, supporting the hyperperfusion model of altered central hemodynamics in this group of pregnant women. A reduction in the PSV value in mild PE is hereby proposed as a mechanism for the characteristic elevated peak ratio observed in patients with $\mathrm{PE}$, while a marginal increase in the PSV value in severe PE compared with mild PE is postulated as the reason for the insignificant change in peak 
ratio between these groups. This study also found the RI to be particularly useful for discriminating mild from severe PE, while the peak ratio, PDV, and EDV were found to help in detection of PE. Overall, this study has shown that OAD ultrasonography is a cheap, noninvasive, readily available, safe, and reliable tool for evaluation of the central hemodynamic changes in normal pregnancy, and even more so in PE.

\section{Acknowledgments}

We thank the staff and resident doctors in the ultrasound suite of the Department of Radiology and booking clinic in the Department of Obstetrics and Gynecology, UCH, Ibadan, for their assistance and cooperation.

\section{Author contributions}

The research was designed by RBO, AJA, MOO, and OAR. RBO performed the Doppler ultrasound studies and along with AJA carried out the data collection. TOO did the data analysis. All authors took part in either drafting the article or revising it critically for important intellectual content. All authors read and approved the final manuscript.

\section{Disclosure}

The authors report no conflicts of interest in this work.

\section{References}

1. Osungbade KO, Ige OK. Public health perspectives of pre-eclampsia in developing countries: implication for health system strengthening. J Pregnancy. 2011;2001:481095.

2. Tamas P, Hantosi E, Bodis J. Hypertensive disorders of pregnancy: theory of hypoperfusion and hyperperfusion types of pre-eclampsia. Gynaecol Perinatol. 2010;19:255-260.

3. World Health Organization. Global program to conquer pre-eclampsia/ eclampsia, 2002. Available from: http://www.ncbi.nlm.nih.gov/pmc/ articles/PMC3087154/. Accessed June 18, 2015.

4. Shah A, Fawole B, M' Imunya JM, et al. Cesarean delivery outcomes from the WHO global survey on maternal and perinatal health in Africa. Int J Gynaecol Obstet. 2009;107:191-197.

5. McClure EM, Saleem S, Pasha O, Goldenberg RL. Stillbirth in developing countries: a review of causes, risk factors and prevention strategies. J Matern Fetal Neonatal Med. 2009;22:183-190.

6. Omole-Ohonsi A, Ashimi AO. Pre-eclampsia: a study of risk factors. Niger Med Pract. 2008;53:99-102.

7. Olopade FE, Lawoyin TO. Maternal mortality in a Nigerian Maternity Hospital. African Journal of Biomedical Research. 2008;11:267-273.

8. Myatt L, Miodovnik M. Prediction of pre-eclampsia. Semin Perinatol. 1999;23:45-57.

9. Oian P, Kjeldsen SE, Eide IK. Pre-eclampsia: a state of sympathetic overactivity. N Engl J Med. 1997;336:1326-1327.

10. Saleh AA, Bottoms SF, Farag AM, et al. Markers for endothelial injury, clotting and platelet activation in pre-eclampsia. Arch Gynecol Obstet. 1992;251:105-110.

11. Schroeder BM. ACOG practice bulletin on diagnosing and managing pre-eclampsia and eclampsia. Am Fam Physician. 2002;66:330-331.

12. Carneiro RS, Sass N, Diniz AL, Souza EV, Torloni MR, Moron AF. Ophthalmic artery Doppler velocimetry in healthy pregnancy. Int $J$ Gynecol Obstet. 2008;100:211-215.
13. Bosio PM, McKenna PJ, Conroy R, O’Herlihy C. Maternal central hemodynamics in hypertensive disorders of pregnancy. Obstet Gynecol. 1999;94:978-984.

14. Lieb WE, Cohen SM, Merton DA, Shields JA, Mitchell DG, Goldberg BB. Color Doppler imaging of the eye and orbit. Arch Ophthalmol. 1991;103:527-531.

15. Jaffe G, Schatz H. Ocular manifestations of pre-eclampsia. Am J Ophthalmol. 1987;103:309-315.

16. Ayaz T, Akansel G, Hayirlioglu A, Arslan A, Suer N, Kuru I. Ophthalmic artery color Doppler ultrasonography in mild-to-moderate pre-eclampsia. Eur J Radiol. 2003;46:244-249.

17. Diniz AL, Moron AF, Santos MC, Sass N. Color Doppler velocimetry of orbital vessels: technique and normal vascular anatomy. Radiol Bras. 2004;37:287-290.

18. Nakatsuka M, Takata M, Tada K, Kudo T. Effect of a nitric oxide donor on the ophthalmic artery flow velocity waveform in pre-eclamptic women. J Ultrasound Med. 2002;21:309-313.

19. Hata T, Senoh D, Hata K, Kitao M. Ophthalmic artery velocimetry in pregnant women. Lancet. 1992;340:182-183.

20. Mackenzie F, De Vermette R, Nimrod C, Boisvert D, Jackson B. Doppler sonographic studies on the ophthalmic and central retinal arteries in the gravid woman. J Ultrasound Med. 1995;14: 643-647.

21. Hata T, Senoh D, Hata K, Kitao M. Ophthalmic artery velocimetry in pre-eclampsia. Gynecol Obstet Invest. 1995;40:32-35.

22. Belfort MA. Doppler assessment of retinal blood flow velocity during parenteral magnesium treatment in patients with pre-eclampsia. Magnes Res. 1993;6:239-246.

23. Diniz AL, Moron AF, Santos MC, Sass N, Pires CR, Debs CL. Ophthalmic artery Doppler as a measure of severe pre-eclampsia. Int $J$ Gynaecol Obstet. 2008;100:216-220.

24. Gurgel Alves JA, Brennecke SP, da Silva Costa F. First trimester triple vascular test for pre-eclampsia prediction. Pregnancy Hypertens. 2012;2:226.

25. Kasiulevivius V, Sapoka V, Filipaviviute R. Sample size calculation in epidemiological studies. Gerontologija. 2006;7:225-231.

26. Correa-Silva EP, Surita FG, Barbieri C, Morais SS, Cecatti JG. Reference values for Doppler velocimetry of the ophthalmic and central retinal arteries in low-risk pregnancy. Int J Gynaecol Obstet. 2012;117:251-256.

27. Walsh CA, Baxi LV. Mean arterial pressure and prediction of preeclampsia. BMJ. 2008;336:1079-1080.

28. Adedapo KS, Olayemi O, Odukogbe AO, Aimakhu CO, Kehinde AO, Salako BL. Increased oxidative stress in Nigerian women with preeclampsia. S Afr J Obstet Gynaecol. 2011;17:50.

29. Adeniji AO, Oparinde DP. Comparison of lipid peroxidation and antioxidant activities in pre-eclamptic and normal pregnancies in Nigerian population. Int J Clin Med. 2013;4:239-243.

30. Idogun ES, Imarengiaye CO, Momoh SM. Extracellular calcium and magnesium in pre-eclampsia and eclampsia. Afr J Reprod Health. 2007;11:89-94.

31. Ananth CV, Keyes KM, Wapner RJ. Pre-eclampsia rates in the United States, 1980-2010: age-period-cohort analysis. BMJ. 2013;347: f6546.

32. Ohno Y, Kawai M, Wakahara Y, Kitagawa T, Kakihara M, Arii Y. Ophthalmic artery velocimetry in normotensive and pre-eclamptic women with or without photophobia. Obstet Gynecol. 1999;94:361-363.

33. Takata M, Nakatsuka M, Kudo T. Differential blood flow in uterine, ophthalmic, and brachial arteries of pre-eclamptic women. Obstet Gynecol. 2002;100:931-938.

34. de Oliveira CA, Moreira de Sa RA, Velarde LGC, da Silva FC, do Vale FA, Netto HC. Change in ophthalmic artery Doppler indices in hypertensive disorders during pregnancy. $J$ Ultrasound Med. 2013;32:609-616.

35. Lisonkova S, Joseph KS. Incidence of pre-eclampsia: risk factors and outcomes associated with early-versus late-onset disease. Am J Obstet Gynecol. 2013;209:544.e1-544.e12. 
36. Ugwu E, Dim CC, Okonkwo CD, Nwankwo TO. Maternal and perinatal outcome of severe pre-eclampsia in Enugu, Nigeria after introduction of magnesium sulphate. Niger J Clin Pract. 2011;14:418-421.

37. Baxter GM, Williamson TH. Color Doppler imaging of the eye: normal ranges, reproducibility, and observer variation. J Ultrasound Med. 1995;14:91-96.

38. Belfort MA, Giannina G, Herd JA. Transcranial and orbital Doppler ultrasound in normal pregnancy and preeclampsia. Clin Obstet Gynecol. 1999;42:479-506.

39. Hata T, Hata K, Moritake K. Maternal ophthalmic artery Doppler velocimetry in normotensive pregnancies and pregnancies complicated by hypertensive disorders. Am J Obstet Gynecol. 1997;177:174-178.

40. Belfort MA, Saade GR, Grunewald C, Dildy GA, Varner MA, Nisell H. Effects of blood pressure on orbital and middle cerebral artery resistances in healthy pregnant women and women with preeclampsia. Am J Obstet Gynecol. 1999;180:601-607.
41. Kuo DM, Chiu TH, Hsieh TT. Maternal renal artery Doppler flow velocity waveform in pre-eclampsia: a preliminary report. $J$ Reprod Med. 1993;38:189-192.

42. Onaran Z, Bilgili YK, Yilmazbas P. Retrobulbar hemodynamics during healthy and pre-eclamptic pregnancy. Gazi Med J. 2012;23:55-58.

43. de Oliveira CA, de Sá RA, Velarde LG, Marchiori E, Netto HC, Ville Y. Doppler velocimetry of the ophthalmic artery in normal pregnancy: reference values. J Ultrasound Med. 2009;28:563-569.

44. Barbosa AS, Pereira AK, Reis ZSN, Lage EM, Leite HV, Cabral ACV. Ophthalmic artery-resistive index and evidence of over perfusion-related encephalopathy in severe preeclampsia. Hypertension. 2010;55:189-193.

45. Cnossen JS, Vollebregt KC, de Vrieze N, et al. Accuracy of mean arterial pressure and blood pressure measurements in predicting preeclampsia: systematic review and meta-analysis. BMJ. 2008;336:1117-1120.

\section{Publish your work in this journal}

The International Journal of Women's Health is an international, peerreviewed open-access journal publishing original research, reports, editorials, reviews and commentaries on all aspects of women's healthcare including gynecology, obstetrics, and breast cancer. The manuscript management system is completely online and includes

\section{Dovepress}

a very quick and fair peer-review system, which is all easy to use. Visit http://www.dovepress.com/testimonials.php to read real quotes from published authors. 